\title{
Maximum Likelihood Detection with Ligand Receptors for Diffusion-Based Molecular Communications in Internet of Bio-Nano Things
}

\author{
Murat Kuscu, Student Member, IEEE and Ozgur B. Akan, Fellow, IEEE
}

\begin{abstract}
Molecular Communication (MC) is a bio-inspired communication technique that uses molecules as a method of information transfer among nanoscale devices. MC receiver is an essential component having profound impact on the communication system performance. However, the interaction of the receiver with information bearing molecules has been usually oversimplified in modeling the reception process and developing signal detection techniques. In this paper, we focus on the signal detection problem of $\mathrm{MC}$ receivers employing receptor molecules to infer the transmitted messages encoded into the concentration of molecules, i.e., ligands. Exploiting the observable characteristics of ligand-receptor binding reaction, we first introduce a Maximum Likelihood (ML) detection method based on instantaneous receptor occupation ratio, as aligned with the current $\mathrm{MC}$ literature. Then, we propose a novel ML detection technique, which exploits the amount of time the receptors stay unbound in an observation time window. A comprehensive analysis is carried out to compare the performance of the detectors in terms of bit error probability (BEP). In evaluating the detection performance, emphasis is given to the receptor saturation problem resulting from the accumulation of messenger molecules at the receiver as a consequence of intersymbol interference (ISI). The results reveal that detection based on receptor unbound time is quite reliable even in saturation, whereas the reliability of detection based on receptor occupation ratio substantially decreases as the receiver gets saturated. Lastly, we also discuss the potential methods of implementing the detectors.
\end{abstract}

Index Terms-Molecular communication, receiver, ligand receptors, maximum-likelihood estimation, signal detection.

\section{INTRODUCTION}

$\mathbf{I}$ NTERNET of Bio-Nano Things (IoBNT) is a novel framework, in which nanomachines and biological entities, such as nanobiosensors, engineered bacteria, are connected with each other and with macroscale networks, e.g., the Internet, to collaboratively enable unprecedented applications, such as continuous health monitoring [1], [2]. Molecular Communications (MC), where molecules are used to encode, transmit and receive information, has emerged as the most promising nanoscale communication paradigm for enabling IoBNT, as it is already widely utilized by living cells [3].

The authors are with the Internet of Everything (IoE) Group, Electrical Engineering Division, Department of Engineering, University of Cambridge, UK (e-mail: \{mk959, oba21\}@cam.ac.uk).

Ozgur B. Akan is also with the Next-generation and Wireless Communications Laboratory (NWCL), Department of Electrical and Electronics Engineering, Koc University, Istanbul, 34450, Turkey (email: akan@ku.edu.tr).

This work was supported in part by the ERC project MINERVA (ERC2013-CoG \#616922), and the EU project CIRCLE (EU-H2020-FET-Open \#665564).
MC has been extensively studied from various aspects. The literature includes channel models, modulation schemes and communication protocols, designed and tailored to the peculiarities of nanoscale and molecular physics and limited capabilities of bio-nano things [4]-[6]. A vast majority of these studies focus on a particular type of MC, where information is encoded into the concentration of molecules, and messages are transferred through diffusion in fluid environment [7]. Detection of concentration-encoded messages is a fundamental problem, which has increasingly attracted the attention of researchers [8], [9]. Living cells sense molecular concentrations through their receptor proteins, called ligand receptors, which can be located at the cell surface or inside the cell and chemically interact with the ligands in their vicinity [10]. In this regard, the receptors constitute an interface between the external environment and internal networks of living cells. On the other hand, current studies focusing on synthetic MC mostly neglect the dynamics of receptors, by assuming that the receiver is a perfect observer capable of counting every single information molecule inside a virtually-defined receiver volume. However, the idealization of the receiver renders all of these approaches impractical; thus, leaves a substantial gap between the theory and practice in every aspects of MC.

This paper focuses on the detection problem for MC receivers with ligand receptors. Ligands, referring to information molecules in this context, interact with receptors by randomly and reversibly binding them. The receptor-ligand interaction can be described by a two-state Markov process governed by the binding and unbinding rates of the ligand-receptor pair [11]. Binding rate is proportional to the ligand concentration in the vicinity of the receptors, while the unbinding rate is invariant to the concentration of ligands. For receptors with a single binding unit, the states of the MC process correspond to the bound and unbound states of the receptors. Finite number of receptors makes the overall interaction nonlinear, which leads to receiver saturation problem when the receptors are exposed to a high concentration of ligands, degrading the sensitivity of the receptors. The existence of receptors complicates the MC detection problem, but at the same time, it unveils a whole new set of observable parameters that are informative of the transmitted symbols.

In this paper, we investigate the feasibility of Maximum Likelihood (ML) detection based on ligand-receptor interaction, and analyze two different detection techniques varying in its practicality and performance. The first method relies on the instantaneous receptor occupation states and uses the ratio 
of bound receptors to decide on the transmitted symbol. This method is quite aligned with the MC studies that utilize ligand receptors at the receiver [11], [12]. We then propose another detection method, which takes a radically different approach and infers the transmitted symbol from the amount of time the receptors stay unbound.

The likelihood ratio tests are formulated for both type of ML detectors by taking into account the intersymbol interference (ISI) resulting from the memory of the diffusion channel. A comprehensive numerical analysis is conducted to compare their performances in different conditions in terms of resulting bit error probability (BEP). For the first time in the MC literature, we elaborate on the receptor saturation problem, and show that detection based on receptor unbound times is quite reliable in the saturation regime of the receiver. We also discuss the practicality of the detectors in light of state-of-theart approaches to devise bio-nanomachines.

The remainder of the paper is organized as follows. In Section II, we review the related work and highlight the main contributions of this study. In Section III, we investigate two different ML estimation schemes which are based on receptor occupation ratio and total receptor unbound time. Details of the considered MC scenario are presented in Section IV. We introduce the ML detectors in Section V. Performance evaluation results are provided in Section VI. In Section VII, we present a brief discussion on the implementation of the detectors. Finally, the concluding remarks are given in Section VIII.

\section{RELATED WORK}

Molecular communication, being the most promising paradigm to enable nanonetworks, has attracted researchers from several disciplines. The literature includes a large number of studies that propose modulation schemes, channel models, advanced communication protocols, applications and transceiver designs tailored to the unique characteristics of this communication method. The interested reader is referred to comprehensive surveys covering various aspects of MC [3], [13], [14].

Regarding the receiver for diffusion-based MC with concentration shift keying modulation, several designs are considered. A vast majority of studies assume that the receiver is a transparent instrument capable of perfectly counting every single molecule in an arbitrarily defined space. Several optimal detector designs, including one-shot and sequence detectors, are proposed for this type of receiver [8], [9], [15], [16]. Recently, there emerged a different approach, which assumes that the receiver counts and absorbs every molecule that hit to its surface [17], [18]. Although the physical relevance for these types of receiver designs is not completely clear yet, they are being frequently employed in MC research to simplify the modeling and analysis.

A bio-inspired and more realistic approach to the reception problem is taken by a couple of studies in synthetic MC literature, which integrate the reversible kinetics of ligand receptors into their models. A noise model for the ligand receptors is introduced in [11]. Capacity analysis for $\mathrm{MC}$ systems with ligand receptors is provided in [12] assuming steady-state conditions for the ligand-receptor interaction. A queueing theory based model for ligand receptors is developed in [19]. Recently, two similar deterministic MC channel models are introduced [20], [21], which take into account the ligand-receptor dynamics. Lastly, we developed a physical model for a SiNW bioFET-based MC receiver, which utilizes ligand receptors as the bio-cyber interface [22], [4]. However, none of these studies accounting for the ligand-receptor binding reactions are focused on developing signal detection techniques for $\mathrm{MC}$ receivers.

For sensory systems with ligand receptors, more has been done in biophysics literature from an estimation theoretical perspective. Beginning from the seminal work of Berg and Purcell [10], many efforts are directed toward understanding the fundamental limits of cellular sensing through ligand receptors under varying conditions, such as those resulting from the fluctuating concentration of ligands and the existence of interfering molecules in the environment [23], [24]. Of particular interest is the proposal of an ML concentration estimation method for a sensory system with single receptor based on the unbound time intervals of the receptor [25]. A very recent and comprehensive review of these efforts is presented in [26].

Detection problem for $\mathrm{MC}$ receivers with ligand receptors has been recently elaborated in [27], where authors develop a Maximum A Posteriori (MAP) decoding scheme that relies on the continuous history of the receptor occupancy states, including the time instances of binding events, to decode the transmitted binary symbol. The scheme is built upon an integrated channel-receiver model which takes the propagation and receptor-ligand binding reaction as a continuous-time Markov process (CTMP) defined on a volume divided into voxels. However, the model is only practical for small-scale systems comprising of a few number of ligands and receptors located in a small volume, and the performance of the proposed detector can be measured only through extensive stochastic simulations of the model.

Compared to [27], this paper takes a more straightforward approach to the detection problem with ligand receptors by applying steady-state assumption on the received ligand concentration based on the difference in the timescales of ligand diffusion and receptor reaction kinetics. We first investigate the performance of an ML detector based on the instantaneous occupation ratio of the receptors, which is more aligned with the current research in the MC literature [11], [12]. Then, we propose a novel detection method based on receptor unbound times. This is inspired from [25], which proposes the use of continuous history of receptor unbound times for ML estimation of ligand concentration. In that regard, it is also similar to the work [27], but the detector proposed in this paper is solely based on receptor unbound times, providing a much more easier design for implementation in resourcelimited bio-nanomachines. One of the main contributions of this paper is to reveal the receptor saturation problem resulting from the ISI for the first time in MC literature, and show that it can be overcome by performing the detection based on the receptors' unbound times instead of their occupation ratio. 


\section{MAXimum LiKelihood Estimation OF Ligand CONCENTRATION}

In this section, we investigate two different ML estimators, which constitute the bases for the detectors introduced in Section V. We consider a scenario, where a sensory system comprising of ligand receptors estimates the ligand concentration based on the observable parameters of ligand-receptor interaction. The receptors are assumed to be exposed to a single type of ligands with a stationary concentration.

In ligand-receptor binding reaction, receptors randomly bind to ligands in their vicinity. A receptor can be either in the Bound (B) or Unbound (U) state. Neglecting the ligand diffusion effects on the reaction with the assumption that the diffusion kinetics of ligands to the receptors are comparably faster than the ligand-receptor binding kinetics, and assuming that there is no interaction, e.g., cooperativity, between receptors, which are exposed to the same concentration of ligands, the state of a single receptor is governed by the following two state Continuous-Time Markov Process (CTMP), i.e.,

$$
\mathrm{U} \underset{k_{-}}{\stackrel{c k_{+}}{\rightleftharpoons}} \mathrm{B},
$$

where $c$ denotes ligand concentration in the vicinity of receptors, $k_{+}$and $k_{-}$are the binding and unbinding rates for the ligand-receptor pair [28]. Note that the overall binding rate, i.e., transition rate from unbound (U) to bound (B) state, is modulated by the ligand concentration $c$.

\section{A. Estimation based on Receptor Occupation Ratio (EROR)}

Assuming steady-state conditions for the ligand-receptor binding reaction, the probability of finding a receptor in the bound state (B) at any time is given by

$$
p_{B}=\frac{c}{c+K_{D}},
$$

where $K_{D}=k_{-} / k_{+}$is the dissociation constant, which governs the affinity between the ligand-receptor pair [29]. The state of a single receptor at steady-state can be considered as a Bernoulli random variable with probability of success $p_{B}$. When the sensory system is composed of $N_{R}$ receptors, the random number of bound receptors $N$ follows Binomial distribution, i.e.,

$$
P\left(N \mid p_{B}\right)=\left(\begin{array}{c}
N_{R} \\
N
\end{array}\right) p_{B}^{N}\left(1-p_{B}\right)^{N_{R}-N},
$$

and the mean number of bound receptors becomes $\mathrm{E}[N]=$ $p_{B} N_{R}$. Equation (3) and the deterministic relation between $p_{B}$ and $c$, given in (2), can be exploited to estimate the concentration $c$ from the observed number of bound receptors $N$. Accordingly, the system can acquire the state of each receptor at once at a specific sampling time, as illustrated in Fig. 1, and use only this information to estimate the concentration. An unbiased estimator for $p_{B}$ can be given by

$$
\hat{p}_{B}=\frac{N}{N_{R}},
$$

and the variance of this estimator is

$$
\sigma_{\hat{p}_{B}}^{2}=\frac{\operatorname{Var}[N]}{N_{R}^{2}}=\frac{p_{B}\left(1-p_{B}\right)}{N_{R}} .
$$

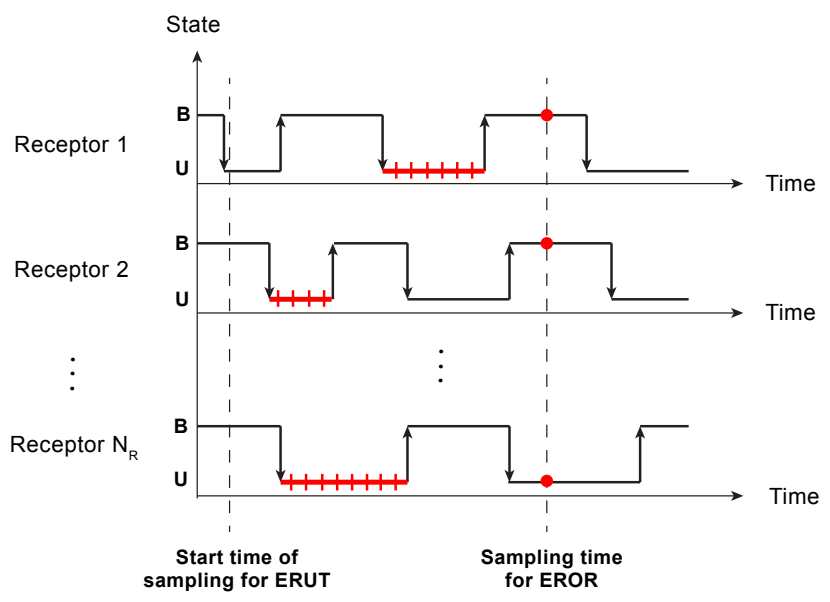

Fig. 1. Sampling for EROR and ERUT schemes, demonstrated over a typical binding sequence of $N_{R}$ receptors.

An estimator for the concentration can then be given by inverting the input-output relation between $c$ and $p_{B}$ in (2), i.e.,

$$
\hat{c}_{N}=K_{D} \frac{\hat{p}_{B}}{1-\hat{p}_{B}} .
$$

Finally, using the rules of error propagation [26], we can write the fractional error in the estimate of concentration as

$$
\begin{aligned}
\left(\frac{\delta \hat{c}_{N}}{c}\right)^{2} & =\frac{1}{c^{2}}\left(\frac{d c}{d p_{B}}\right)^{2} \sigma_{\hat{p}_{B}}^{2} \\
& =\frac{1}{p_{B}\left(1-p_{B}\right) N_{R}} .
\end{aligned}
$$

Note that the estimation error approaches infinity as occupation ratio $p_{B}$ nears 0 or 1 , corresponding to sparsely occupied and saturation regimes of the sensory system, respectively.

\section{B. Estimation based on Receptor Unbound Time (ERUT)}

Receptors undergo a series of binding and unbinding events when exposed to a ligand concentration, and thus a series of independent bound and unbound time intervals can be observed. The amounts of bound and unbound times follow exponential distributions with the corresponding rate parameters $\lambda_{b}=k_{-}$ and $\lambda_{u}=c k_{+}$, respectively. This implies that the receptors at the bound state are insensitive to the ligand concentration $c$, and only the unbound time intervals are informative of the concentration.

For the concentration estimation, we consider that the receiver takes a single sample of unbound time interval from each receptor. From practical point of view, this corresponds to a scenario, where the receiver triggers its receptors at a predefined time, highlighted as the start time of sampling in Fig. 1, to inform about the length of the first unbound interval just after the next unbinding event. The corresponding unbound time intervals are also demonstrated in Fig. 1. Hence, the receiver is assumed to collect $N_{R}$ number of independent samples for the length of receptor unbound times from $N_{R}$ number of receptors. The time necessary for collecting this 


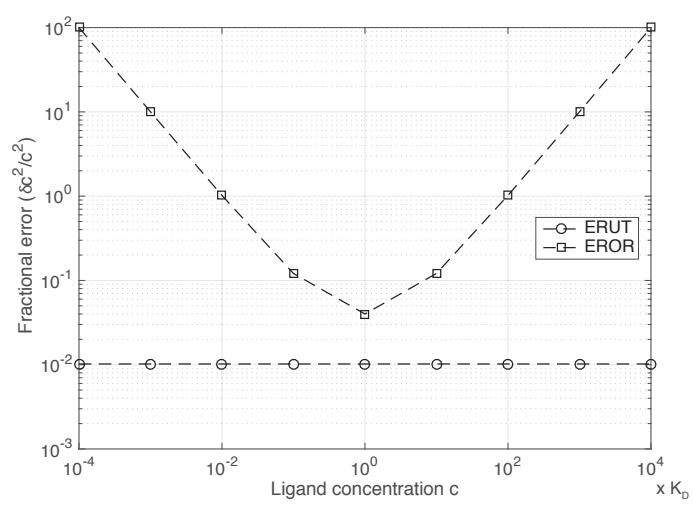

(a)

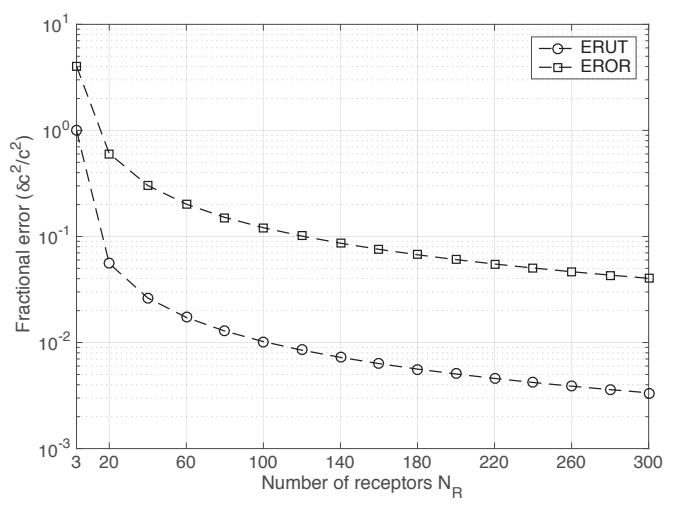

(b)

Fig. 2. Fractional errors in concentration estimates based on EROR and ERUT schemes (a) with varying concentration $c$, and (b) with varying number of receptors $N_{R}$

information can be approximated by considering the worstcase scenario, where a receptor is triggered just after it gets into the unbound state, as demonstrated in the trajectory of Receptor 1 in Fig. 1. In this case, it takes an amount of time corresponding to two unbound and one bound intervals for the sampling to be completed. Therefore, the average of maximum sampling time $\tau_{s}$ can be given by

$$
\mu_{\tau_{s}}=2 \mu_{\tau_{u}}+\mu_{\tau_{b}}
$$

where $\mu_{\tau_{u}}=1 / \lambda_{u}=1 /\left(k_{+} c\right)$ and $\mu_{\tau_{b}}=1 / \lambda_{b}=1 / k_{-}$are the mean unbound and bound time intervals, respectively.

In light of above assumption, the likelihood of observing a particular set of $N_{R}$ unbound time intervals is given by

$$
f\left(\left\{\tau_{u}\right\} \mid c\right)=\prod_{i=1}^{N_{R}} k_{+} c e^{-k_{+} c \tau_{u, i}}=e^{-k_{+} c T_{U}}\left(k_{+} c\right)^{N_{R}},
$$

where $\left\{\tau_{u}\right\}=\left\{\tau_{u, 1}, \ldots \tau_{u, i}, \ldots \tau_{u, N_{R}}\right\}$ is the set of unbound time intervals observed on $N_{R}$ receptors with $\tau_{u, i}$ denoting the length of $i^{\text {th }}$ unbound time interval, and $T_{U}=\sum_{i=1}^{N_{R}} \tau_{u, i}$ is the total length of stay in the unbound state. The log-likelihood of observing a set of unbound time intervals is then written as

$$
\mathcal{L}_{T}\left(c ;\left\{\tau_{u}\right\}\right)=\ln f\left(\left\{\tau_{u}\right\} \mid c\right)=N_{R} \ln \left(k_{+} c\right)-k_{+} c T_{U} .
$$

If we equate the derivative of the log-likelihood function in (10) with respect to $c$ to zero, we obtain an expression for the ML estimate of ligand concentration, i.e., $c_{T}^{*}$,

$$
\begin{gathered}
\frac{\partial \mathcal{L}_{T}}{\partial c}=\frac{N_{R}}{c}-k_{+} T_{U}=0, \\
\hat{c}_{T}^{*}=\frac{N_{R}}{k_{+} T_{U}} .
\end{gathered}
$$

As being the sum of $N_{R}$ independent and identically distributed (i.i.d.) exponential random variables $\tau_{u}$, mean of which is $1 /\left(k_{+} c\right), T_{U}$ becomes a gamma distributed random variable, i.e., $f\left(T_{U} \mid c\right)=\operatorname{Gamma}\left(N_{R}, k_{+} c\right)$, with the mean and variance given by

$$
\begin{aligned}
\mathrm{E}\left(T_{U} \mid c\right) & =\frac{N_{R}}{k_{+} c}, \\
\operatorname{Var}\left(T_{U} \mid c\right) & =\frac{N_{R}}{\left(k_{+} c\right)^{2}} .
\end{aligned}
$$

On the other hand, the reciprocal of the total unbound time of receptors, i.e., $1 / T_{U}$, follows inverse gamma distribution, with mean $\left(k_{+} c\right) /\left(N_{R}-1\right)$. Therefore, the mean of the estimator $\hat{c}_{T}^{*}$ is given as follows

$$
\mathrm{E}\left[\hat{c}_{T}^{*}\right]=\frac{c N_{R}}{N_{R}-1},
$$

which suggests that the estimator is biased. As hinted by (15), an unbiased estimator can be given by

$$
\hat{c}_{T}=\frac{N_{R}-1}{k_{+} T_{U}},
$$

and the fractional error of this unbiased estimator can be written as

$$
\begin{aligned}
\left(\frac{\delta \hat{c}_{T}}{c}\right)^{2} & =\frac{1}{c^{2}}\left(\frac{N_{R}-1}{k_{+}}\right)^{2} \operatorname{Var}\left(\frac{1}{T_{U}}\right) \\
& =\frac{1}{N_{R}-2} \text { for } N_{R}>2 .
\end{aligned}
$$

In the second line of (17), we use the fact that $1 / T_{U}$ is inverse gamma distributed, and its variance is given by $\operatorname{Var}\left(1 / T_{U}\right)=$ $\left(k_{+} c\right)^{2} /\left(\left(N_{R}-1\right)^{2}\left(N_{R}-2\right)\right)$ for $N>2$.

Comparing ERUT and EROR schemes in terms of fractional errors in their estimation, i.e., comparing (7) and (17), we see that while the error in EROR estimate approaches infinity as the mean occupation ratio $p_{B}$ gets close to 0 or 1 , the extent of error for ERUT is invariant to this ratio, hinting at a substantial performance improvement in saturated and sparsely occupied regimes of the sensory system.

\section{Comparison of ML Estimators}

Here, we numerically compare the performance of the estimators in terms of lower bounds on the estimation errors in Fig. 2. The results, when $N_{R}=100$, and $c$ varies between 
$10^{-4} K_{D}$ and $10^{4} K_{D}$, are presented in Fig. 2(a). As is seen, the performance of the first estimator substantially degrades when the system is exposed to a very low or very high concentration, i.e., when it is sparsely occupied or almost saturated. On the other hand, the second estimator based on total unbound time is invariant to ligand concentration, and always performs better than the first estimator.

In the second analysis, the ligand concentration is kept constant at $c=10 K_{D}$, while the number of observations is varied. As is seen in Fig. 2(b), the estimation error decreases with increasing number of observations, and the ERUT-based estimator surpasses the EROR-based estimator by a factor of $1 /\left(p_{B}\left(1-p_{B}\right)\right)$, which goes to infinity as the occupation ratio approaches 0 or 1 .

In summary, the ERUT-based estimator has a substantial advantage over the EROR-based estimator especially when the system is sparsely occupied $\left(p_{B} \simeq 0\right)$ or saturated $\left(p_{B} \simeq\right.$ $1)$. In the following, we introduce the ML detectors for MC built upon these estimators, and compare their performances in terms of bit error probability.

\section{Communication System Model}

We consider a time-slotted molecular communication system between a single transmitter nanomachine (Tx) and a single receiver nanomachine $(\mathrm{Rx})$ in a three dimensional fluid environment, which has infinite extent in all dimensions. Tx and Rx are assumed to be synchronized with each other in terms of time. The system utilizes ON/OFF keying (OOK) modulation such that the point source Tx transmits $Q$ molecules as an impulse at the beginning of a time slot to represent the input symbol $s=1$, and does not transmit any molecule during the time slot to represent $s=0$.

Transmitted molecules freely propagate in the channel through diffusion, and some of them achieve to reach the $\mathrm{Rx}$ location. For the reception process, we follow the same assumptions in [11]. Accordingly, we assume that the receptors are homogenously distributed inside the receiver volume, and the boundaries of the receiver has negligible effect on the transport dynamics of ligands. Considering that the distance between the Tx and Rx is expected to be very large compared to the dimensions of the nanomachines, we assume that ligand concentration is also homogenous inside the receiver volume and equal to its value at the receiver location. Solving the Fick's second law of diffusion for free propagation of ligands, the channel impulse response is obtained as [9]

$$
h(t)=(4 \pi D t)^{-3 / 2} \exp \left(-\frac{d^{2}}{4 D t}\right),
$$

where $d$ is the Tx-Rx distance, and $D$ is the diffusion coefficient. Then, the concentration at the receiver location during the $i^{\text {th }}$ signaling interval becomes

$$
c_{[i]}(t)=\sum_{j=i-I}^{i} s_{[j]} Q h\left(t+(i-j) T_{s}\right) \text { for } t \in\left[0, T_{s}\right),
$$

where $Q$ is the number of transmitted molecules, $T_{s}$ is the symbol interval, $s_{[j]} \in\{0,1\}$ is the transmitted symbol in the $j^{t h}$ signaling interval, and $I$ is the channel memory length, i.e., number of interfering symbols. In fact, $I$ goes to infinity in a molecular communication channel, as there is always nonzero probability to find a previously transmitted molecule in the receiver volume. However, this probability quickly decreases with time; thus, the problem here is simplified by considering a finite $I$.

\section{Maximum Likelihood Detection with Ligand RECEPTORS}

Given the system model in Section IV, the detection problem can be cast as a binary hypothesis testing problem, which is formulated for the $i$ th signaling interval as follows

$$
\begin{aligned}
& H_{1}: Q \text { molecules emitted at } t=(i-1) T_{s} \text {, i.e., } s_{[i]}=1 \\
& H_{0}: \text { No molecule emitted at } t=(i-1) T_{s} \text {, i.e., } s_{[i]}=0
\end{aligned}
$$

The ML detection methods introduced in the sequel aim at solving the above binary hypotesis testing problem based on the ligand-receptor interaction parameters observable by the receiver.

In developing the detection methods, following the work in [16], we assume that the receiver has a finite memory, and it keeps $M$ number of previously decoded bits in its memory to make use of them along with the channel impulse response function (18) to estimate the interference resulting from previous transmissions. Given that the sampling time is fixed and equal to $t_{s}$ for any signaling interval, we can write the ISI estimate of receiver in the $i^{t h}$ signaling interval as

$$
\begin{aligned}
\Omega_{[i]} & =\sum_{j=i-M}^{i-1} \hat{s}_{[j]} Q h\left(t_{s}+(i-j) T_{s}\right) \\
& +p_{1} Q \sum_{j=i-I}^{i-M-1} h\left(t_{s}+(i-j) T_{s}\right),
\end{aligned}
$$

where $\hat{s}_{[i]}$ is the $i^{t h}$ decoded bit, and $p_{1}=P\left\{s_{[i]}=1\right\}$ is the probability for the transmitter to transmit bit-1, which is taken as equal to 0.5 , i.e., events $s_{[i]}=1$ and $s_{[i]}=0$ are equiprobable. Here, the first summand in the RHS of (21) is the estimated value of ISI resulting from $M$ most recent bits decoded by the receiver. The last summand of (21) is the ISI resulting from the $(I-M)$ number of bits transmitted prior to the $M$ most recent ones, which is taken as equal to its mean.

\section{A. Detection based on Receptor Occupation Ratio (DROR)}

Using the estimation method described in Section III-A, we now develop a detection technique based on the instantaneous occupation ratio of the receptors. As we discussed earlier, receiver makes use of the number of bound receptors at the sampling time together with its channel impulse response and previously decoded bits stored in its memory to decide between the hypotheses $H_{0}$ and $H_{1}$.

The estimated likelihood of observing $N$ bound receptors at the sampling time, conditioned on the current transmitted 
symbol $s_{[i]}=\{0,1\}$ and receiver's ISI estimate $\Omega_{[i]}$ can be written by using (2), (3) and (21), i.e.,

$$
\begin{aligned}
P^{*}\left(N \mid s_{[i]}, \Omega_{[i]}\right)=\left(\begin{array}{c}
N_{R} \\
N
\end{array}\right) & \left(\frac{\Omega_{[i]}+s_{[i]} \Phi}{\Omega_{[i]}+s_{[i]} \Phi+K_{D}}\right)^{N} \\
& \times\left(\frac{K_{D}}{\Omega_{[i]}+s_{[i]} \Phi+K_{D}}\right)^{N_{R}-N},
\end{aligned}
$$

where $\Phi=Q h\left(t_{s}\right)$, and superscript ${ }^{*}$ denotes that this is an estimated likelihood function. Based on (22), the hypothesis testing problem can be simplified to a likelihood ratio test (LRT), i.e.,

$$
\frac{P^{*}\left(N \mid s_{[i]}=1, \Omega_{[i]}\right)}{P^{*}\left(N \mid s_{[i]}=0, \Omega_{[i]}\right)} \underset{H_{0}}{\stackrel{H_{1}}{\gtrless}} 1 .
$$

The LRT can be further simplified by introducing a detection threshold $\lambda_{D R O R,[i]}$, i.e.,

$$
N \underset{H_{0}}{\stackrel{H_{1}}{\gtrless}} \lambda_{D R O R,[i]}=N_{R} \frac{\ln \left(\frac{\Omega_{[i]}+\Phi+K_{D}}{\Omega_{[i]}+K_{D}}\right)}{\ln \left(\frac{\Omega_{[i]}+\Phi}{\Omega_{[i]}}\right)},
$$

where the receiver decides $H_{1}$ when $N=\lambda_{D R O R,[i]}$.

\section{B. Detection Based on Receptor Unbound Time (DRUT)}

As revealed in Section III-B, ligand concentration at the receiver location can be inferred from the total unbound time of receptors $T_{U}$, when the number of observed unbound intervals are known to the receiver. Here, we propose a novel detection method based on this estimation scheme. The receiver is assumed to acquire the knowledge of $N_{R}$ unbound time intervals from its receptors, and use this observation for decoding the transmitted symbol $s$. We also assume that during the observation time window, the ligand concentration at the receiver location remains stationary. This assumption is frequently utilized in MC literature [11], [12], and largely holds true because the transmitted signals are low-pass filtered by the diffusion process, making the time-scale of biologicallyrelevant ligand-receptor binding reactions is very low compared to the timescale of deviations in the ligand concentration. Additionally, the amount of time required for obtaining $N_{R}$ samples is on the scale of time required for just two successive unbinding-binding events in a single receptor (see (8), and Appendix A for further discussion). Under this assumption, the estimated probability density of the total unbound time of receptors conditioned on the current transmitted bit $s_{[i]}$ and estimated ISI $\Omega_{[i]}$ can be written as

$$
\begin{aligned}
& f^{*}\left(T_{U} \mid s_{[i]}, \Omega_{[i]}\right) \\
& =\frac{\left(k_{+}\left(\Omega_{[i]}+s_{[i]} \Phi\right)\right)^{N_{R}}}{\Gamma\left(N_{R}\right)} T_{U}^{N_{R}-1} e^{-k_{+}\left(\Omega_{[i]}+s_{[i]} \Phi\right) T_{U}}
\end{aligned}
$$

where $\Gamma($.$) is the complete gamma function. The log-$ likelihood is then given by

$$
\begin{aligned}
& \mathcal{L}_{U}^{*}\left(T_{U} ; s_{[i]}, \Omega_{[i]}\right)=\ln f^{*}\left(T_{U} \mid s_{[i]}, \Omega_{[i]}\right) \\
& =N_{R} \ln \left[k_{+}\left(\Omega_{[i]}+s_{[i]} \Phi\right)\right]-k_{+} T_{U}\left(\Omega_{[i]}+s_{[i]} \Phi\right)+C,
\end{aligned}
$$

where $C$ is a constant term representing the summands that are invariant to $s_{[i]}$ and cancelled out in the likelihood-ratio test, which is formulated as follows

$$
\mathcal{L}_{U}^{*}\left(T_{U} ; s_{[i]}=0, \Omega_{[i]}\right) \underset{H_{1}}{\stackrel{H_{0}}{\gtrless}} \mathcal{L}_{U}^{*}\left(T_{U} ; s_{[i]}=1, \Omega_{[i]}\right)
$$

The test is further simplified by introducing a time-varying threshold variable $\lambda_{D R U T,[i]}$, i.e.,

$$
T_{U} \underset{H_{1}}{\stackrel{H_{0}}{\gtrless}} \lambda_{D R U T,[i]}=\frac{N_{R}}{k_{+} \Phi} \ln \left(\frac{\Omega_{[i]}+\Phi}{\Omega_{[i]}}\right)
$$

It can be inferred from the detection rule (28) that higher unbound times are more likely to result from $s=0$ transmission, i.e., implying less amount of ligands at the receiver. When the concentration becomes higher, there is a higher chance that an unbound receptor quickly rebinds to another ligand.

\section{PERFormance EVAluation}

We carried out a comparative analysis to evaluate the performance of the introduced detection schemes in terms of resulting bit error probabilities (BEPs) under different system settings with varying parameters, such as memory length $M$, number of transmitted molecules $Q, \mathrm{Tx}-\mathrm{Rx}$ distance $d$. The default values of system parameters used in the analysis are given in Table I, and the selection criteria for them are discussed in Appendix A. We apply Monte Carlo method to estimate the corresponding BEPs as the average value resulting from 1000 runs of simulation of transmitting a pseudorandom sequence of 1000 bits for each different system setting. In each run of the Monte Carlo simulation, BEP values are computed recursively using the detection thresholds given in (24) and (28).

To increase the signal-to-interference ratio (SIR), we assume that the receiver samples the receptor states or triggers the receptors to inform about the unbound time when the ligand concentration at the receiver location attains its maximum for $s=1$ transmission, i.e. $t_{s}=t_{\text {peak }}$. Peak time can be derived from the impulse response (18), and depends only on the Tx-Rx distance and diffusion coefficient, i.e., $t_{\text {peak }}=\frac{d^{2}}{6 D}$. Additionally, we arbitrarily set the default signaling interval to $T_{S}=4 \times t_{\text {peak }}$, which leads to a moderate ISI in default setting.

Throughout the analysis, along with the results in terms of $\mathrm{BEP}$, we also provide plots for typical occupation ratio $p_{B}^{*}$ as function of the system parameter under evaluation to show the degree of saturation and sparsity, and its effect on the detector

TABLE I

Default Values of Simulation Parameters

\begin{tabular}{l|l}
\hline \hline Number of transmitted ligands for $s=1(Q)$ & $5 \times 10^{7}$ \\
\hline Transmitter-receiver distance $(d)$ & $10 \mu \mathrm{m}$ \\
\hline Diffusion coefficient of ligands $(D)$ & $1 \times 10^{-10} \mathrm{~m}^{2} / \mathrm{s}$ \\
\hline Binding rate $\left(k_{+}\right)$ & $2 \times 10^{-19} \mathrm{~m}^{3} / \mathrm{s}$ \\
\hline Unbinding rate $\left(k_{-}\right)$ & $20 \mathrm{~s}$ \\
\hline Number of receptors on the receiver surface $\left(N_{R}\right)$ & 100 \\
\hline ISI length $(I)$ & 25 \\
\hline Receiver memory length $(M)$ & 2 \\
\hline Symbol interval $\left(T_{S}\right)$ & $4 \times t_{\text {peak }}$ \\
\hline Sampling time $\left(t_{s}\right)$ & $t_{\text {peak }}$ \\
\hline
\end{tabular}




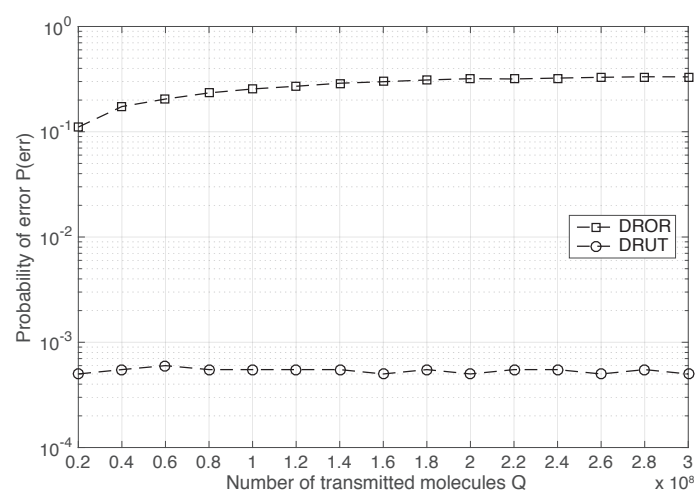

(a)

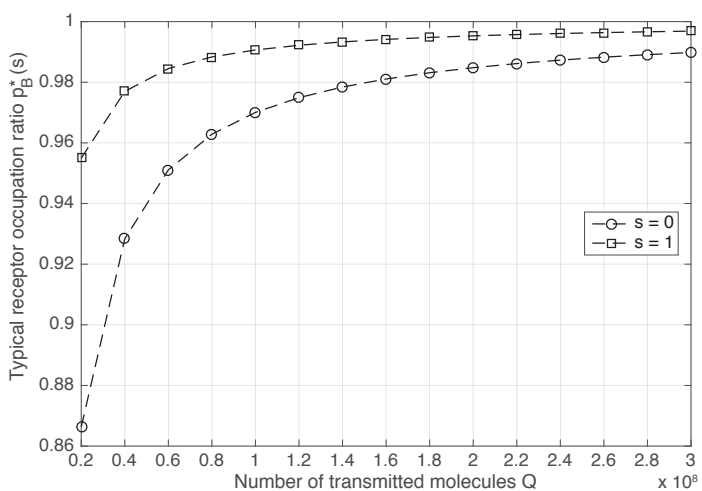

(b)

Fig. 3. Effect of number of transmitted molecules $Q$ on the detection performance: (a) bit error probability $P(e r r)$ with varying $Q$, (b) corresponding typical receptor occupation ratio $p_{B}^{*}$.

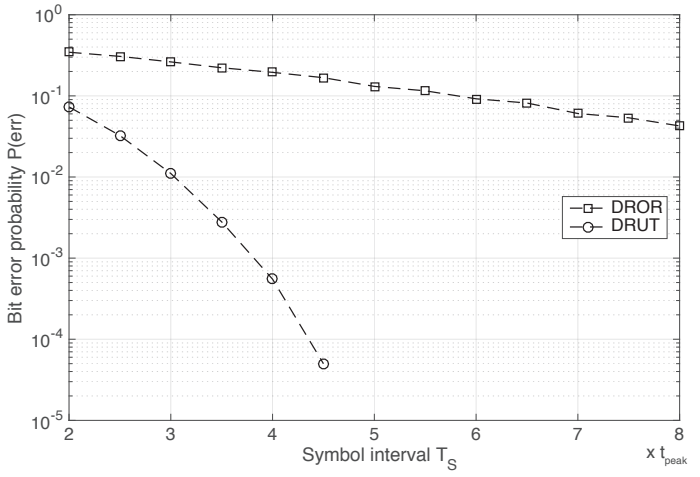

(a)

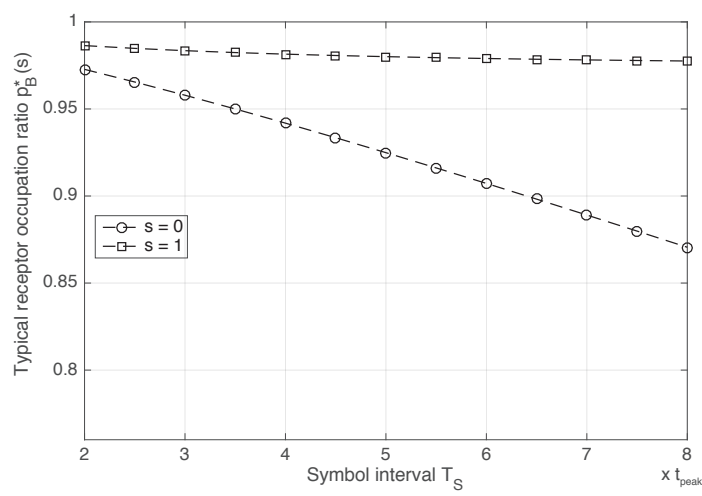

(b)

Fig. 4. Effect of symbol interval length $T_{S}$ on the detection performance: (a) bit error probability $P($ err $)$ with varying $T_{S}$, (b) corresponding typical receptor occupation ratio $p_{B}^{*}$.

performance. It is calculated as the mean receptor occupation ratio corresponding to the expected value of concentration at the receiver volume, i.e.,

$$
p_{B}^{*}(s)=\frac{\mathrm{E}[c \mid s]}{\mathrm{E}[c \mid s]+K_{D}},
$$

where $\mathrm{E}[c \mid s]=s Q h\left(t_{s}\right)+p_{1} Q \sum_{j=1}^{I} h\left(t_{s}+j T_{S}\right)$ is the mean ligand concentration at the receiver volume conditioned on the transmitted bit in the current signaling interval, with $s=\{0,1\}$ denoting the bit 0 or bit 1 transmission.

\section{A. Effect of Number of Transmitted Molecules}

We first investigate the effect of number of transmitted molecules $Q$ on the detection performance. As $Q$ increases, more ligands reach the receiver, making the receptors approach the saturation, as is evident from the varying typical receptor occupation ratio plotted in Fig. 3(b). As the receptors get saturated, the performance of the DROR-type detector is severely degraded. BEP increases to almost 0.5 , implying that the detector becomes unable to discriminate the transmitted binary symbols from each other. On the other hand, as seen in Fig. 3(a), the performance of the DRUT-type detector is not affected by this parameter. The reason may not be obvious at first sight. As we see in (13) and (14), the mean and standard deviation of the total unbound time of receptors are scaled with the same factor as the concentration is varied. Therefore, DRUT-type detector, which is based on receptor unbound time, has the same performance regardless of the received concentration and the number of transmitted molecules. Furthermore, note that the DRUT-type detector always outperforms the DROR-type detector, independent of $Q$.

\section{B. Effect of Signaling Interval}

In the second analysis, we study the relation between the length of signaling intervals $T_{S}$, i.e., the reciprocal of the transmission rate, and the detection performance. Decreasing the length of signaling interval, i.e., increasing the transmission rate, leads to the saturation of the receptors by increasing the ISI and the ligand concentration at the receiver. However, the increase in the concentration with decreasing $T_{S}$ does not occur at the same rate for $s=0$ and $s=1$ transmission as captured in (19) and evident from Fig. 4(b). Therefore, different from the first analysis, we see that both type of detectors are substantially affected by the length of signaling intervals. As seen in Fig. 4(a), the advantage of DRUTtype detector becomes more evident as the transmission rate 


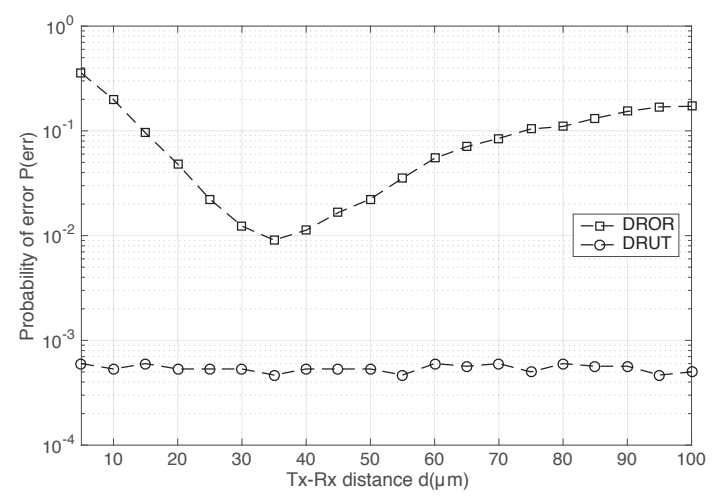

(a)

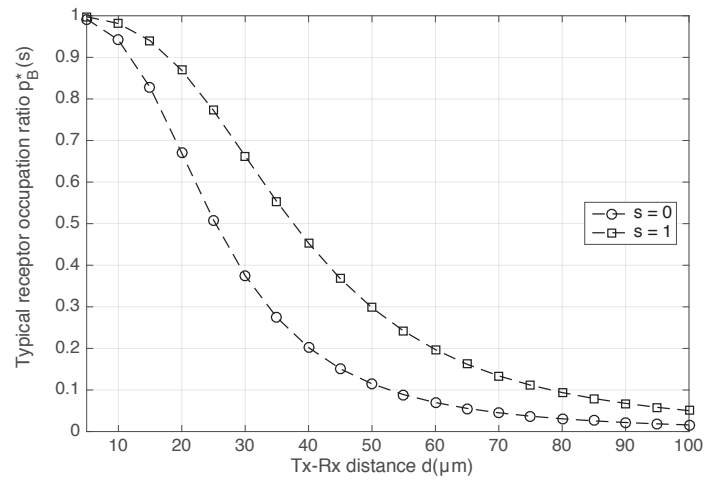

(b)

Fig. 5. Effect of Tx-Rx distance $d$ on the detection performance: (a) bit error probability $P(e r r)$ with varying $d$, (b) corresponding typical receptor occupation ratio $p_{B}^{*}$.

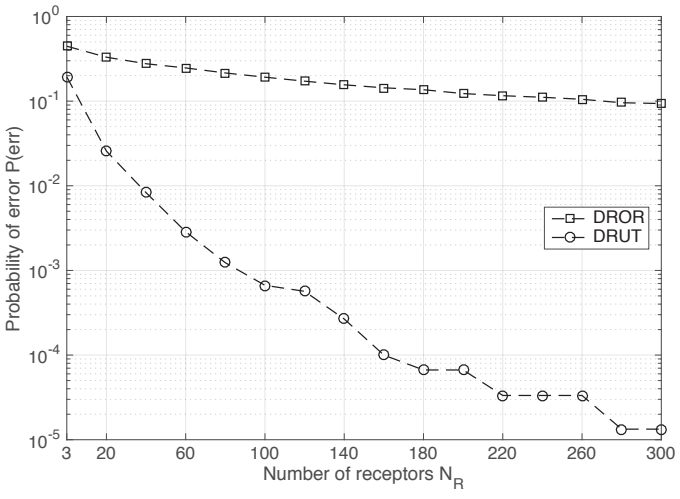

(a)

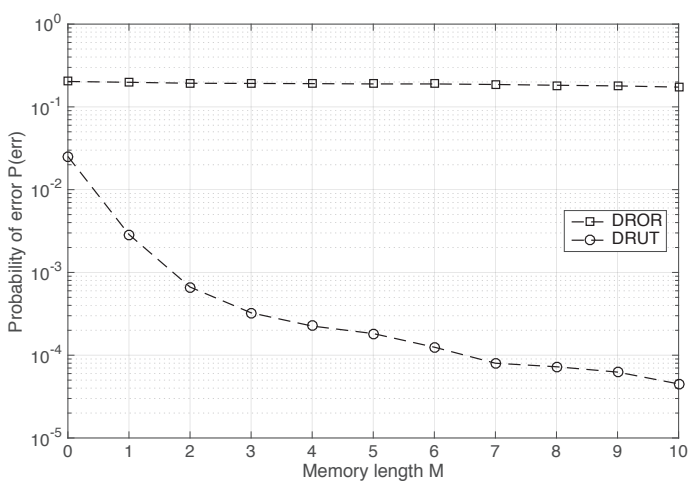

(b)

Fig. 6. Bit error probability $P(e r r)$ with (a) varying number of receptors $N_{R}$, and (b) varying receiver memory length $M$.

decreases.

\section{Effect of Distance}

Next, we analyze the detection performance with varying Tx-Rx distance $d$. As the distance increases while $Q$ is held constant, concentration at the receiver decreases for both $s=0$ and $s=1$ transmissions. As seen in Fig. 5(b), the typical occupation ratio ranges between values corresponding to sparsely occupied and saturated receiver, while the distance is increased from $10 \mu \mathrm{m}$ to hundred $100 \mu \mathrm{m}$. The DROR scheme performs poorly under both saturation and sparsity conditions, when the typical occupation ratios for $s=0$ and $s=1$ approach to each other, and it becomes harder for the receiver to discriminate the two symbols. However, the performance of DRUT-type detector is not affected by the distance, due to exactly the same reasons as we explain in the first analysis in Section VI-A.

\section{Effect of Number of Receptors}

We perform another analysis to evaluate the effect of varying number of receptors $N_{R}$ available in the reception space, which is equal to the number of samples used for detection. Default parameter values given in Table I are set to make the receptors almost saturate for both $s=1$ and $s=0$. Under this conditions, the result of the analysis is shown in Fig. 6(a). As is seen, the performance of both type of detectors improves with increasing number of receptors. However, the performance improvement in the DRUT-type detector is more evident than the DROR-type detector. The difference can be attributed to the significantly different extent of errors resulting from the underlying estimation methods especially under saturation conditions, which has been covered in Section III.

Note that our model does not reflect the effect of spatial dimensions of the reception space and thus the receptor concentration, and it is based on the assumption that the receptors operate independently of each other. However, high concentration of receptors on a sensory system can give rise to correlations between receptors, due to the correlated transport of ligands to the closely located receptors. The resulting correlation between receptors is found to have a small and negative effect on the ability of sensory system to estimate the ligand concentration [32]. However, it is not included in this study for the sake of mathematical tractability. 


\section{E. Effect of Receiver Memory}

The last analysis is performed to reveal the effect of varying length of receiver memory $M$ on the detection performance. As is seen in Fig. 6(b), increasing the memory length significantly improves the performance of the DRUT-type detector, whereas only a slight decrease in the probability of error is observed for DROR-type detector. This analysis once again signifies that DRUT-type detector substantially outperforms the DROR-type detector regardless of system parameters.

\section{DisCUSSION ON IMPLEMENTATION}

In this section, we briefly review the introduced detectors from a practical point of view and discuss the implementation challenges. There are basically two approaches to implement bio-nanomachines operating in molecular nanonetworks. The first one is based on the use of synthetic nanomaterials, such as SiNW and graphene, as the building blocks of the nanomachines. For example, a few studies recently proposed the use of SiNW FET-based nanoscale biosensors (BioFETs), which provide affinity-based detection based on ligand-receptor binding mechanism, as the MC receivers [4], [22], [33]. However, in bioFET-based receivers, the sensory information received from ligand receptors is converted, in a combined manner, into an electrical signal contaminated by various noise sources at the output. Therefore, observing the state of individual receptors is not possible with this technology.

The other approach, which is based on synthetic biology, is to implement transmission and reception within living cells by modifying the natural gene circuits or creating new synthetic ones [34]. The technology is already mature enough to allow performing complex digital computations, e.g., with networks of genetic NAND and NOR gates, as well as analog computations, such as logarithmically linear addition, ratiometric and power-law computations, in synthetic cells [35]. Synthetic gene networks integrating computation and memory is also proven feasible [36]. More importantly in this context, the technology enables implementing bio-nanomachines capable of observing individual receptors, as naturally done by living cells; thus, stands as a more suitable domain for practically implementing the introduced detectors.

The acquisition of information about the ligand concentration starts with the binding of receptors to ligands. The transduction of the ligand concentration signal to an intracellular molecular signal is realized through downstream signaling networks within the receiver cell. This transduction network outputs a kind of readout molecules inside the cells, which can directly control or modulate a cell activity, or can be an input to another intracellular signaling network, which further processes the data before modulating a cell activity. The latter is the case with the detection mechanisms proposed in this paper, as another signaling network will be necessary for decoding the received signals. The state-of-the-art synthetic biology techniques now enable the design of new kinds of receptor-ligand interactions giving rise to new functionalities for receptors and signaling networks [37], [38]. Basically, the conformational changes upon interaction and chemical activation of receptors are the main control parameters for designing receptors with new functionalities [39].
Different network designs can be utilized to implement a cell network, which can achieve the acquisition of the receptor states for both detection schemes. Let us consider the following intracellular reaction network for the DROR scheme, in which the receiver cell needs to sample the instantaneous binding states of the receptors at a given sampling time:

$$
\begin{gathered}
S+R^{B} \stackrel{k_{S_{+}^{*}}^{\longrightarrow}}{\longrightarrow} R^{B}+S^{*}, \\
S^{*} \stackrel{k_{S_{-}^{*}}^{\longrightarrow}}{\longrightarrow} S .
\end{gathered}
$$

Here, $R^{B}, S$ and $S^{*}$ denote the bound receptors, readout molecules and activated readout molecules, respectively. Assuming that the reaction rates $k_{S_{+}^{*}}$ and $k_{S_{-}^{*}}$ are very high compared to the ligand-receptor binding reaction rates, the concentration of activated readout molecules $S^{*}$ becomes modulated by the instantaneous number of bound receptors. In this way, the intracellular concentration of $S^{*}$ at the sampling time can be utilized as input to another reaction network within cell to carry out the proposed decoding operation.

The acquisition of unbound time intervals for the DRUT scheme requires more sophisticated reaction networks, as it should include the triggering of the receptors to start reporting the unbound state. The following reaction network can be given as an example for the signal transduction in this scheme:

$$
\begin{gathered}
\varnothing \stackrel{k_{A+}}{\stackrel{k_{A-}}{\longrightarrow}} A, \\
R_{I}^{B}+A \stackrel{k_{A}}{\longrightarrow} R_{A}^{B}+A, \\
R_{I}^{U}+A \stackrel{k_{A}}{\longrightarrow} R_{A^{*}}^{U}+A, \\
R_{A^{*}}^{U}+L \stackrel{k_{+}}{\longrightarrow} R_{A}^{B}, \\
R_{A}^{B} \stackrel{k_{-}}{\longrightarrow} R_{A}^{U}+L, \\
R_{A}^{U}+L \stackrel{k_{+}}{\longrightarrow} R_{I}^{B}, \\
R_{A}^{U}+S \stackrel{k_{M}}{\longrightarrow} R_{A}^{U}+S^{*} .
\end{gathered}
$$

Here, the receptors $R$ have five different states, only one of which is able to react with the readout molecules $S$. Superscripts $B$ and $U$ denote the bound and unbound states of the receptors, respectively, and subscripts $I, A^{*}$, and $A$ denote the inactive, semi-active, and active states of the receptors, respectively. There are also activator molecules $A$, which are produced with rate $k_{A+}$ when triggered by the receiver at the start time of the sampling for each signaling interval, and degrades with rate $k_{A-}$. In this network, only the activated unbound receptors $R_{A}^{U}$ react with the readout molecules $S$ producing activated readout molecules $S^{*}$ as the product with rate $k_{S}$. Therefore, the concentration of the active readout molecules is modulated by the total unbound time interval $T_{U}$, thus they can be used as input to an intracellular decoding network implementing DRUT scheme. The other reactions governing the activation and deactivation of receptors in bound and unbound states, given in (33)-(37), ensure the proper acquisition of the unbound time data according to the sampling scheme illustrated in Fig. 1. Note that the generation and degradation rates of activator molecules $k_{A+}$ and $k_{A-}$ should be high compared to the ligand-receptor binding reaction rates 
to ensure that the inactivated receptors are not re-activated during the same signaling interval. It is also to be noted that the reaction (38) is a quantization operation, which encodes the continuous unbound time information into discrete number of activated readout molecules $S^{*}$; therefore, the production rate of $S^{*}$, i.e., $k_{S}$, determines the extent of the quantization noise, i.e., the higher the rate, the lower the noise.

The detectors investigated in this paper work based on the likelihood ratio test principle. A comparator gene circuit capable of converting an input analog signal into a binary signal with an adjustable threshold would be sufficient for the bio-nanomachine to realize any of the detection methods. A recent study has proposed a genetic comparator circuit integrating analog and digital computation functionalities, which could serve the needs of the introduced detectors [40]. For DROR and DRUT schemes, the sensory information, which is either the sum of instantaneous receptor occupation states at the sampling time or the total unbound time of receptors, can be directly fed into the analog input of the comparator in terms of concentration of readout molecules activated by the receptors. The threshold level of the comparator, however, must be set through a series of arithmetic operations on the mean of the ligand concentration at the receiver's vicinity around the sampling time and the previously detected symbols stored in the receiver's memory, see (24), (28).

The complexity of the required arithmetic operations would add to the detection error, since each computation in a gene network brings its own uncertainty. In addition, for DRUT type detector, the representation of the analog unbound times with discrete readout molecules would introduce further quantization errors which could propagate to the final readout of the comparator. Fortunately, the quantization errors could be overcome by sufficiently increasing the activation rate of readout molecules by the receptors.

The intrinsic noise in genetic networks, potential errors in the estimation of parameters required for the comparator, and stability issues regarding the memory all add to the overall uncertainty and contribute to the final detection error. However, developing a more comprehensive detection model taking these errors into account is beyond the scope of this study, and thus, remains as a research challenge.

\section{CONCLUSION}

In this paper, we investigate the detection problem in diffusion-based $\mathrm{MC}$ receivers with ligand receptors. Exploiting the characteristics of the ligand-receptor binding interaction, we propose a novel and easy-to-implement detection method which infers the transmitted symbol from the amount of time the receptors stay in the unbound state. The performance of the proposed detection method is compared to a more conventional detection scheme, which relies on the occupation ratio of the receptors. The results, presented in terms of bit error probability, reveal that the proposed method is substantially more effective in overcoming the saturation problem resulting from the ISI intrinsic to the MC channels.

\section{APPENDIX}

\section{A. Signaling Interval for DRUT}

As we see in Section III, there is a tradeoff between the time necessary for the receiver to acquire enough data and the accuracy of the estimation. Although ERUT scheme provides much better estimate of the ligand concentration than the EROR scheme, it takes the receiver longer to collect the necessary information, i.e., receptor unbound times, from the receptors. As given in (8), in the worst case scenario, the average time required to sample a receptor unbound time from each receptor can be expressed by

$$
\mu_{\tau_{s}}=2 \mu_{\tau_{u}}+\mu_{\tau_{b}}=2 /\left(k_{+} c\right)+1 / k_{-},
$$

which shows that the decision can take longer as the ligand concentration at the receiver gets smaller. This poses a challenge for the MC detection problem, as the receiver needs to collect samples and carry out the decoding before another message of transmitter arrives. This could be a problem especially when the transmitter sends successive bit-0's, which in turn makes the ligand concentration at the receiver very low. Therefore, we find it crucial to put a constraint on the signaling interval $T_{s}$, to specify a range for system parameters, for which we can safely assume that the receiver finds enough time to sample the total unbound time of $N_{R}$ receptors in DRUT scheme. To this aim, we consider the worst-case scenario as being the successive transmission of 10 bit-0's, since the probability for the transmitter to successively send bit-0's for more than 10 times gets too small (i.e., $\leq\left(1-p_{1}\right)^{11}$ ) for $p_{1}=0.5$, such that it can be neglected. Accordingly, we write the constraint as follows

$$
\begin{aligned}
T_{s} & >2 \mu_{\tau_{u}}+\mu_{\tau_{b}}, \\
& >\frac{2}{k_{+} p_{1} Q \sum_{i=H}^{\infty} h\left(t_{s}+i T_{s}\right)}+\frac{1}{k_{-}},
\end{aligned}
$$

where $H=10$ is the number of successive bit-0's that have been sent. This constraint is typically satisfied when the receiver is expected to operate near saturation, as the receptor unbound times get shorter; however, other system parameters, such as the unbinding rate $k_{-}$and the distance represented in the channel impulse response $h(t)$, can also affect the validity of the constraint. The range of parameter values, for which we evaluate the performance of the detection schemes in Section VI, readily satisfies this condition, as the operating conditions are set to make the receiver operate near saturation.

\section{REFERENCES}

[1] I. F. Akyildiz, M. Pierobon, S. Balasubramaniam and Y. Koucheryavy, "The Internet of bio-nano things," IEEE Communications Magazine, vol. 53, no. 3, pp. 32-40, 2015.

[2] M. Kuscu and O. B. Akan, "The Internet of molecular things based on FRET," IEEE Internet of Things Journal, vol. 3, no. 1, pp. 4-17, 2016.

[3] O. B. Akan, H. Ramezani, T. Khan, N. A. Abbasi and M. Kuscu, "Fundamentals of molecular information and communication science," Proceedings of the IEEE, vol. 105, no. 2, pp. 306-318, 2017.

[4] M. Kuscu and O. B. Akan, "Modeling and analysis of SiNW FET-based molecular communication receiver," IEEE Transactions on Communications, vol. 64, no. 9, pp. 3708-3721, 2016.

[5] I. F. Akyildiz et al., "Monaco: Fundamentals of molecular nanocommunication networks," IEEE Wireless Comm., vol. 19, no. 5, pp. 12-18, 2012. 
[6] T. Nakano, M. J. Moore, F. Wei, A. V. Vasilakos, J. Shuai, "Applications of molecular communications to medicine: A survey," IEEE Trans. Nanobiosci., vol. 11, no. 2, pp. 135-148, 2012.

[7] M. Pierobon and I. F. Akyildiz, "Capacity of a diffusion-based molecular communication system with channel memory and molecular noise," IEEE Trans. Inf. Theory, vol. 59, no. 2, pp. 942-954, 2013.

[8] D. Kilinc and O. B. Akan, "Receiver design for molecular communication," IEEE J. Sel. Areas Commun., vol. 31, no. 12, pp. 705-714, 2013.

[9] L.-S. Meng, P.-C. Yeh, K.-C. Chen and I. F. Akyildiz, "On receiver design for diffusion-based molecular communication," IEEE Trans. Signal Process., vol. 62, no. 22, pp. 6032-6044, 2014.

[10] H.C. Berg and E.M. Purcell, "Physics of chemoreception," Biophys. J., vol. 20, pp. 193-219, 1977.

[11] M. Pierobon and I. F. Akyildiz, "Noise analysis in ligand-binding reception for molecular communication in nanonetworks," IEEE Trans. Signal Process., vol. 59, no. 9, pp. 4168-4182, 2011.

[12] G. Aminian, M. Farahnak-Ghazani, M. Mirmohseni, M. Nasiri-Kenari and Faramarz Fekri, "On the capacity of point-to-point and multipleaccess molecular communications with ligand-receptors," IEEE Trans. Mol. Biol. Multi-Scale Commun., vol. 1, no. 4, pp. 331-346, 2015.

[13] L. Felicettia, M. Femminellaa, G. Realia, and P. Lio, "Applications of molecular communications to medicine: A survey," Nano Commun. Net. J., vol. 7, pp. 27-45, 2016.

[14] T. Nakano, T. Suda, Y. Okaie, M. J. Moore and A. V. Vasilakos, "Molecular communication among biological nanomachines: A layered architecture and research issues," IEEE Trans. Nanobiosci., vol. 13, no. 3, pp. 169-197, 2014.

[15] A. Noel, K. C. Cheung and R. Schober, "Optimal receiver design for diffusive molecular communication with flow and additive noise," IEEE Trans. Nanobiosci., vol. 13, no. 3, pp. 350-362, 2014.

[16] R. Mosayebi, H. Arjmandi, A. Gohari, M. Nasiri-Kenari and U. Mitra, "Receivers for Diffusion-Based Molecular Communication: Exploiting Memory and Sampling Rate," IEEE J. Sel. Areas Commun., vol. 32, no. 12, pp. 2368-2380, 2014

[17] H. B. Yilmaz, A. C. Heren, T. Tugcu and C.-B. Chae, "ThreeDimensional Channel Characteristics for Molecular Communications With an Absorbing Receiver," IEEE Commun. Lett., vol. 18, no. 6, pp. 929-932, 2014.

[18] M. Damrath, S. Korte and P. Hoeher, "Equivalent Discrete-Time Channel Modeling for Molecular Communication With Emphasize on an Absorbing Receiver," IEEE Trans. Nanobiosci., vol. 16, no. 1, pp. 60-68, 2017.

[19] L. Felicetti, M. Femminella and G. Reali, "A simple and scalable receiver model in molecular communication systems," in Proc. ACM NANOCOM 2016, New York, NY, USA, September 2016.

[20] Y. Deng, A. Noel, M. Elkashlan, A. Nallanathan and K. C. Cheung, "Modeling and simulation of molecular communication systems with a reversible adsorption receiver," IEEE Trans. Mol. Biol. Multi-Scale Commun., vol. 1, no. 4, pp. 347-362, 2015.

[21] A. Ahmadzadeh, H. Arjmandi, A. Burkovski and R. Schober, "Comprehensive reactive receiver modeling for diffusive molecular communication systems: reversible binding, molecule degradation, and finite number of receptors," IEEE Trans. Nanobiosci., vol. 15, no. 7, pp. 713-727, 2016.

[22] M. Kuscu and O. B. Akan, "On the physical design of molecular communication receiver based on nanoscale biosensors," IEEE Sensors J., vol. 16, no. 8, pp. 2228-2243, 2016.

[23] K. Kaizu, W. Ronde, J. Paijmans, K. Takahashi, F. Tostevin and P. R. Wolde, "The Berg-Purcell limit revisited," Biophys. J., vol. 106, no. 4, pp. 976-985, 2014.

[24] W. Bialek and S. Setayeshgar, "Physical limits to biochemical signaling," Proc. Natl. Acad. Sci., vol. 102, no. 29, 2015.

[25] R. G. Endres and N. S. Wingreen, "Maximum likelihood and the single receptor,” Phys. Rev. Lett., vol. 103, no. 158101, 2009.

[26] P. R. Wolde, N. B. Becker, T. E. Ouldridge and A. Mugler, "Fundamental limits to cellular sensing," J. Stat. Phys., vol. 162, no. 5, pp. 13951424 , 2016.

[27] C. T. Chou, "Maximum a-posteriori decoding for diffusion-based molecular communication using analog filters," IEEE Trans. Nanotechnol., vol. 14, no. 6, pp. 1054-1067, 2015.

[28] A. M. Berezhkovskii and A. Szabo, "Effect of ligand diffusion on occupancy fluctuations of cell-surface receptors," J. Chem. Phys., vol. 139, no. 12, p. 121920, 2013.

[29] W. Bialek, Biophysics: Searching for Principles. Princeton, NJ: Princeton University Press, 2012.

[30] S. M. Kay, Fundamentals of Statistical Signal Processing: Estimation Theory, 2nd ed. Upper Saddle River, NJ, USA: Prentice Hall PTR, 1993.

[31] R. B. Ash, Probability and Measure Theory, 2nd ed. New York, NY, USA: Academic, December 1999.
[32] V. Singh, M. Tchernookov and I. Nemenman "Effects of receptor correlations on molecular information transmission," Physical Review E, vol. 94, no. 2, p. 022425, 2016.

[33] M. Kuscu and O. B. Akan, "Modeling and analysis of SiNW bioFET as molecular antenna for bio-cyber interfaces towards the Internet of bionanothings," in Proc. IEEE WF-IoT 2015, Milan, Italy, December 2015.

[34] B. D. Unluturk, A. O. Bicen and I. F. Akyildiz, "Genetically engineered bacteria-based biotransceivers for molecular communication," IEEE Trans. Commun., vol. 63, no. 4, pp. 1271-1281, 2015.

[35] R. Daniel, J. R. Rubens, R. Sarpeshkar and T. K. Lu, "Synthetic analog computation in living cells," Nature, vol. 497, pp. 619-623, 2013.

[36] O. Purcell and T. K Lu, "Synthetic analog and digital circuits for cellular computation and memory," Curr. Opin. Chem. Biol., vol. 29, pp. 146-155, 2014.

[37] L. L. Looger, M. A. Dwyer, J. J. Smith and H. W. Hellinga, "Computational design of receptor and sensor proteins with novel functions,' Nature, vol. 423, pp. 185-190, 2003.

[38] E. Andrianantoandro, S. Basu, D. K. Karig and R. Weiss, "Synthetic biology: new engineering rules for an emerging discipline," Mol. Syst. Biol., vol. 2, no. 1, 2006.

[39] C. Hoffmann, A. Zurn, M. Bunemann and M. J. Lohse, "Conformational changes in G-protein-coupled receptors - the quest for functionally selective conformations is open," Br. J. Pharmacol., vol. 153, no. S1, pp. 358-366, 2008.

[40] J. R. Rubens, G. Selvaggio and T. K. Luu, "Synthetic mixed-signal computation in living cells," Nature Commun., vol. 7, pp. 11658, 2016.

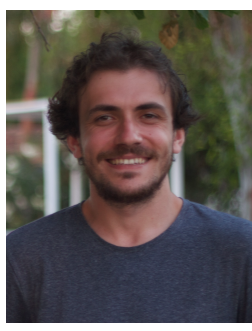

Murat Kuscu [S'11] received his B.Sc. degree in electrical and electronics engineering from Middle East Technical University, Turkey, in 2011, and the M.Sc. and Ph.D. degrees in Electrical and Electronics Engineering Department of Koc University, Turkey, in 2013 and 2017, respectively. He is currently a research assistant in the Internet of Everything (IoE) Group, Electrical Engineering Division, Department of Engineering, University of Cambridge, UK. His current research interests include nanoscale and molecular communications, and

Internet of Everything.

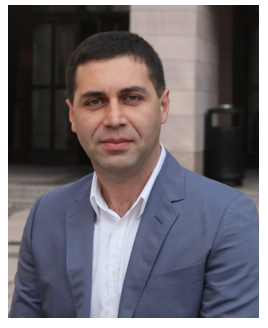

Ozgur B. Akan [M'00-SM'07-F'16] received the Ph.D. degree in electrical and computer engineering from the School of Electrical and Computer Engineering, Broadband and Wireless Networking Laboratory, Georgia Institute of Technology, Atlanta, GA, USA, in 2004. He is currently with the Internet of Everything Group, Electrical Engineering Division, Department of Engineering, University of Cambridge, UK, and also the Director of the Nextgeneration and Wireless Communications Laboratory at Department of Electrical and Electronics Engineering, Koc University, Istanbul, Turkey. His research interests include wireless communications, nano, molecular, and neural communications, and information theory. He is an Associate Editor of the IEEE transactions on communication, the IEEE transactions on vehicular technology, the IET Communications, and the Nano Communication Networks Journal (Elsevier). 